\title{
Cd(Mg)Se Single Layers and CdSe/CdMgSe Heterostructures Grown by Molecular Beam Epitaxy on InAs(001) Substrates
}

\author{
V.A. Kaygorodov, V.S. Sorokin \\ St. Petersburg Electrotechnical University \\ Prof. Popov 5, St. Petersburg 197376, Russia
}

\author{
I.V. Sedova, O.V. Nekrutkina, S.V. Sorokin, \\ T.V. Shubina, A.A. Toropov and S.V. Ivanov \\ Ioffe Physico-Technical Institute of RAS \\ Politekhnicheskaya 26, St. Petersburg 194021, Russia
}

\begin{abstract}
We report on molecular beam epitaxy of CdSe/CdMgSe heterostructures on InAs(001) substrates and studies of their optical and structural properties. The CdMgSe energy gap versus composition dependence is determined. The zinc-blende MgSe band-gap energy and optical bowing parameter are estimated to be $4.05 \mathrm{eV}$ and $0.2 \mathrm{eV}$, respectively. The CdSe quantum wells embedded into CdMgSe barriers demonstrate intense photoluminescence. Effective mass approximation calculations of electron-heavy hole optical transitions in CdSe quantum well are in a good agreement with the experimental data obtained.
\end{abstract}

PACS numbers: 78.66.Hf

\section{Introduction}

Recently, a new approach to designing mid-infrared laser diodes has been proposed, which consists in combining III-V and II-VI compounds in a single-double heterostructure [1]. A CdSe/CdMgSe system is of great importance due to its possibility to be employed as the II-VI part in such In As-based lasers. However, there have been practically no data on growth and properties of this system until 
now. CdSe has been well studied only when inserted in a ZnSe matrix, where due to $7 \%$ lattice mismatch the CdSe sheets transform into broadened up to 10 monolayer (ML) graded-composition ZnCdSe layers with self-organized quantum disk-like Cd composition fluctuations even in a submonolayer range of CdSe thicknesses [2]. It prevents studying of the optical and electronic properties of "pure" cubic CdSe quantum wells (QWs). On the other hand, an InAs/CdSe heteropair is quite suitable for this purpose due to a small lattice mismatch between binaries $(\approx 0.3 \%)$. Moreover, the CdMgSe solid alloy with Mg content of $10 \%$ is lattice matched to In As and can serve as a barrier for the CdSe QW. Further increase in the Mg content allows to grow an alternately strained CdSe/CdMgSe multiple QW structure lattice-matched to InAs as a whole. To our knowledge, the cubic CdMgSe has been never grown epitaxially on InAs(001) substrates so far, although some optical and structural properties of bulk melt-grown hexagonal CdMgSe crystals have been reported [3].

In this paper we present for the first time a complex study of optical and structural properties of low-strained zinc-blende CdSe/Cd(Mg)Se QW structures grown by molecular beam epitaxy (MBE) on InAs(001) substrates.

\section{Experiment}

The structures were grown consequently in two separate MBE chambers. An InAs buffer layer was grown on InAs(001) substrate in a III-V MBE setup (Riber $32 \mathrm{P}$ ), covered with polycrystalline As to protect the surface from oxidation in atmosphere and then transferred to the II-VI MBE growth chamber (home made EP1203) through air. Elemental Cd, Mg, Zn, Te, and Se molecular beam sources were employed for the epitaxy. Before the growth, the As cover was removed by annealing the sample at substrate temperature $T_{\mathrm{S}}=440-480^{\circ} \mathrm{C}$. A reflection high-energy electron diffraction (RHEED) system was used to monitor surface conditions. It has been found that the optimal II-VI growth initiation conditions correspond to an intermediate $(2 \times 4) \mathrm{As} \&(4 \times 2)$ In surface reconstruction achieved at $T_{\mathrm{S}} \approx 460^{\circ} \mathrm{C}$. Two types of growth initiation procedure at the III-V/II-VI heterovalent interfaces were used. During the first one, $1 \mathrm{ML}$ of CdSe was initially grown in MBE mode to prevent an uncontrollable interaction between Se atoms and the InAs surface, on the analogy with the $\mathrm{ZnSe} / \mathrm{GaAs}$ case [4]. Then a $10 \mathrm{~nm}$ thick $\mathrm{CdMgSe}$ layer was grown in $\mathrm{MBE}$ mode at $\approx 200^{\circ} \mathrm{C}$ to reduce the defects density on the In As/CdSe interface [5], which usually resulted in a streaky RHEED patterns. The deposition times of $\mathrm{Cd}$ and $\mathrm{Mg}$ were chosen to provide desirable alloy composition. The following growth of the CdMgSe/CdSe structure occurs at $280^{\circ} \mathrm{C}$ in MBE mode under the $(2 \times 1)$ Se-stabilized conditions. In the other case, $T_{\mathrm{S}}=280^{\circ} \mathrm{C}$ was kept constant from the very beginning of II-VI growth which was initiated with a deposition of $\approx 5 \mathrm{~nm}$ thick ZnTe in the MBE mode. Detailed comparative analysis of the two buffer layer sequences and their effect on 
structural properties of CdMgSe layers will be published elsewhere [6]. The typical structure includes the bottom CdMgSe barrier of 0.3-0.9 $\mu \mathrm{m}$ thick, followed by the CdSe QW of a thickness ranging within 2-6 $\mathrm{nm}$ and capped with a $0.1 \mu \mathrm{m}$ $\mathrm{CdMgSe}$ barrier. Thin CdSe layer was grown on top to prevent a CdMgSe oxidation. Additionally, single CdMgSe layers were grown to study their energy gap optical bowing.

Different techniques including cross-sectional scanning electron microscopy (SEM) and transmission electron microscopy (TEM), electron probe microanalysis (EPMA), X-ray diffraction (XRD) and photoluminescence (PL) were used for structural and optical characterization of the QW structures. XRD rocking curves were measured in a symmetric Bragg geometry with a (004) reflection. A Ge-crystal was used as a monochromator for the $\mathrm{Cu} K_{\alpha}$ radiation. The PL measurements were performed at liquid nitrogen temperature using the $325 \mathrm{~nm}$ line of a cw $\mathrm{He}-\mathrm{Cd}$ laser.

\section{Results and discussion}

Figure 1a presents a cross-section TEM image of the CdSe/CdMgSe QW structure, with the $2 \mathrm{~nm}$ thick CdSe QW being visible as a thin uniform dark stripe. In this case, the ZnTe buffer layer was used to form the heterovalent III-V/II-VI interface. Neither stacking faults nor dislocations are observed in the image, which indicates a perfect structural quality of the structure. The PL spec-

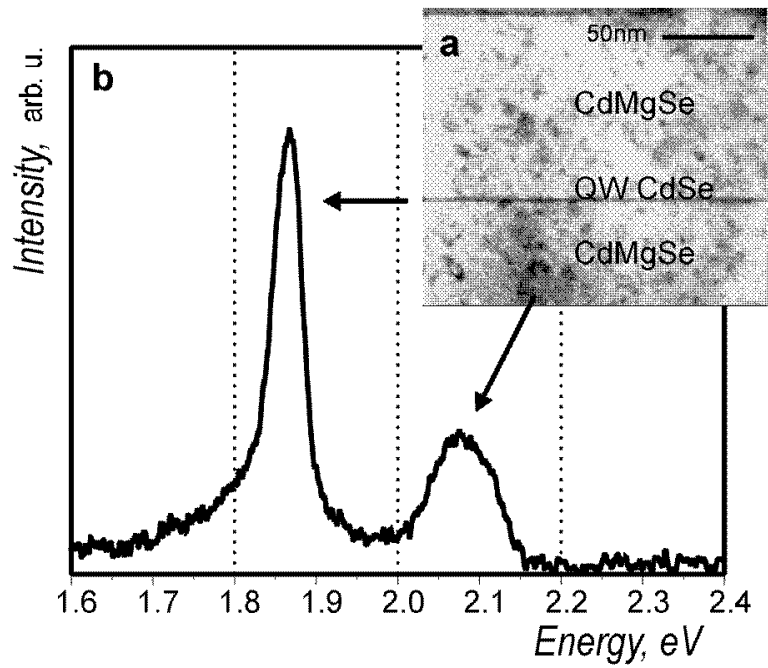

Fig. 1. (a) Cross-sectional TEM image of the CdSe/CdMgSe QW structure, with the $2 \mathrm{~nm}$ thick CdSe QW. The ZnTe buffer layer was used to form the heterovalent III-V/II-VI interface. (b) Low-temperature PL spectrum of the $3.4 \mathrm{~nm}$-CdSe/CdMgSe QW structure. 
trum of $3.4 \mathrm{~nm}-\mathrm{CdSe} \mathrm{QW}$ structure is shown in Fig. 1b. One can clearly see two peaks at 2.077 and $1.868 \mathrm{eV}$, which are attributed to the near-band-edge radiative recombination in the $\mathrm{CdMgSe}$ claddings and optical transitions between electron and hole energy levels in the CdSe QW, respectively. The asymmetric shape of the former peak is likely caused by a possible gradient of $\mathrm{Mg}$ content in the CdMgSe layer. The PL spectrum allows us to determine the energy gap of the corresponding layer as follows:

$$
E_{\mathrm{G}}=h \nu+E_{\mathrm{ex}}+E_{\mathrm{St}} \pm \Delta E_{\mathrm{str}}
$$

where $h \nu$ is the optical transition energy, $E_{\mathrm{ex}}$ is the exciton binding energy, $E_{\mathrm{St}}$ is the Stocks shift, and $\Delta E_{\text {str }}$ is the variation of the energy gap due to the strain effect. The exciton binding energy in $\mathrm{Cd}_{1-x} \mathrm{Mg}_{x} \mathrm{Se}(0<x<0.25)$ is taken as $15 \mathrm{meV}$, which is the same as that of bulk CdSe [7]. The Stocks shift is estimated as a half width at half maximum (HWHM) of the PL peak, while the strain influence on the band structure of CdSe or CdMgSe layer is taken into account according to the "model-solid" theory [8].

Figure 2 demonstrates an XRD $\theta-2 \theta$ rocking curve of a single CdMgSe structure. The joint analysis of the XRD and EPMA data allows us to estimate independently the $\mathrm{Mg}$ content and relaxation degree of the CdMgSe layers as 0.14 and $60 \%$, respectively. For this sample the relaxation degree of the CdMgSe layer is estimated as $\approx 60 \%$, which indicates that the layer thickness is more than critical one. Hence, in this case the strain effect on CdMgSe band-gap energy can be neglected.

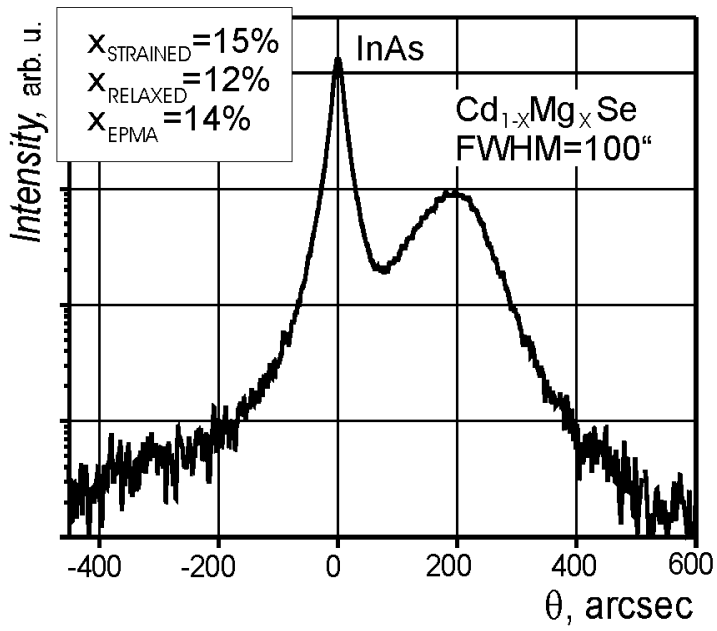

Fig. 2. XRD $\theta-2 \theta$ rocking curve of a single CdMgSe structure. The comparatively large FWHM of the CdMgSe peak seems to be due to possible gradient of Mg content in CdMgSe layer. 
Taking into account the above mentioned factors, we estimate the compositional dependence of the CdMgSe band-gap energy using the following equation (see also Fig. 3):

$$
E_{\mathrm{G}}^{\mathrm{CdMgSe}}(x)=x E_{\mathrm{G}}^{\mathrm{MgSe}}+(1-x) E_{\mathrm{G}}^{\mathrm{CdSe}}-C x(1-x),
$$

where $E_{\mathrm{G}}^{\mathrm{MgSe}}$ and $E_{\mathrm{G}}^{\mathrm{CdSe}}$ are the energy gaps of MgSe and CdSe, respectively; $C$ is the bowing parameter of the CdMgSe alloy. The zinc-blende CdSe band-gap energy has been found from PL of bulk CdSe/InAs layers to be $1.746 \mathrm{eV}$, which is in good agreement with the data reported before [9]. There have been various estimates of the energy gap of "pure" MgSe, ranging from 3.59 to $5.60 \mathrm{eV}[10,11]$. However, as follows from our data the good $E_{\mathrm{G}}$ estimate for zinc-blende $\mathrm{MgSe}$ is about $4.05 \mathrm{eV}$, which coincides with the data of Litz et al. [12]. The fitted curve obtained at $C=0.2 \mathrm{eV}$ seems to be in good agreement with experimental data obtained from the PL spectra of $\mathrm{Cd}_{1-x} \mathrm{Mg}_{x}$ Se layers with different magnesium content $(0<x<0.32)$, which are shown in the inset in Fig. 3. The full width at half maximum (FWHM) of the band-edge PL peaks increases with the Mg content raising, which can be explained by enhanced disordering of the solid alloys.

According to the common-anion rule, the increase in $E_{\mathrm{G}}$ of CdMgSe should be accompanied by moving only the conduction band upwards. However, by the

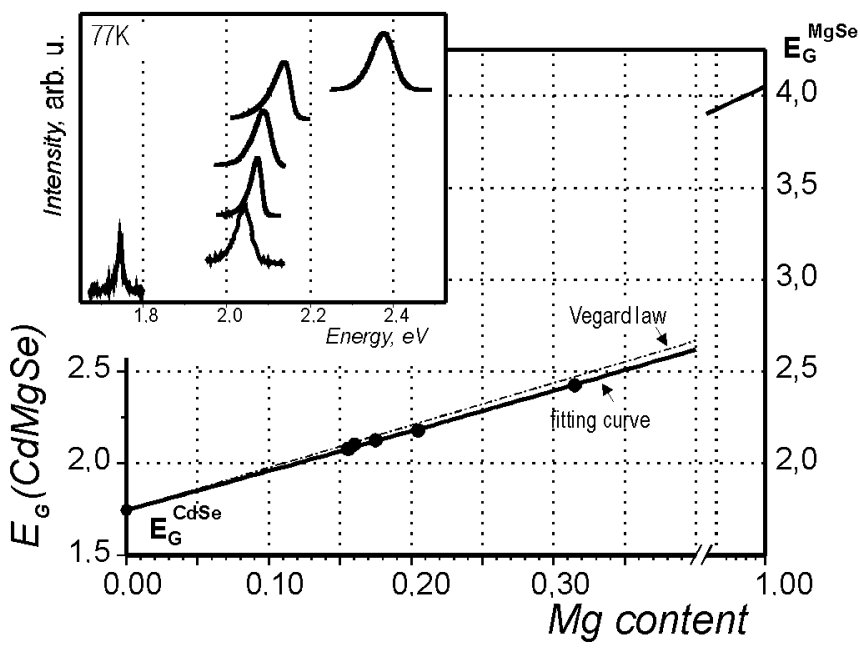

Fig. 3. $E_{\mathrm{G}}$ versus $x$ dependence for the $\mathrm{Cd}_{1-x} \mathrm{Mg}_{x}$ Se system. $E_{\mathrm{G}}$ values and compositions $x$ of CdMgSe alloys were obtained from PL spectra and EPMA data. Circles are the experimental data. Solid curve is a $E_{G}(x)$ theoretical estimation using optical bowing parameter $C=0.2 \mathrm{eV}$. The dashed line shows the fitting according to the Vegard law. Inset shows low-temperature PL spectra of the CdMgSe single layers with different Mg contents $(0<x<0.32)$. 
analogy with the $\mathrm{ZnCdSe} / \mathrm{ZnSe}$ system where the valence band offset (VBO) is not zero in spite of the common anion in both binaries [13], the similar valence band behavior is expected for the CdMgSe/CdSe heterostructures. Theoretical calculations using first-principles all-electron band structure method give the CdSe/MgSe VBO as high as $1.1 \mathrm{eV}$ [14], although this value appears to be too large. Combining the experimental value of $\mathrm{MgSe} / \mathrm{Cd}_{0.56} \mathrm{Zn}_{0.44} \mathrm{Se}$ VBO [15] with the well known $\Delta E_{\mathrm{V}} / \Delta E_{\mathrm{G}}$ ratio of 0.25 for the $\mathrm{ZnCdSe} / \mathrm{CdSe}$ system [13], we have obtained the smaller $\Delta E_{\mathrm{V}} / \Delta E_{\mathrm{G}}$ ratio for $\mathrm{CdSe} / \mathrm{Cd}_{1-x} \mathrm{Mg}_{x}$ Se of about 0.3 (or VBO $=0.66 x \mathrm{eV}$ ). Taking into account strain influence on the band structure, the band offsets at the CdMgSe/CdSe interface for electrons $U_{\mathrm{e}}$ and heavy holes $U_{\mathrm{h}}$ can be determined by analogy with the ZnSe/ZnCdSe QW case [16].

Table shows the experimental PL peak energies of the CdSe/CdMgSe QW structures with different CdSe QW thicknesses and Mg contents in CdMgSe barriers. The respective calculated energies of e1-hh1 transitions, obtained in the effective mass approximation taking into account the strain effects, are also given in Table. We took into consideration the e1-hh1 transition only because the light-hole energy level is well above the heavy-hole one due to significant compressive strain in the CdSe QW. The energies of e1 $\left(\varepsilon_{e}\right)$ and hh1 $\left(\varepsilon_{h h}\right)$ quantum levels are determined as follows:

$$
\sqrt{\frac{m_{\mathrm{CdSe}}}{m_{\mathrm{CdMgSe}}} \frac{U_{\mathrm{e}(\mathrm{hh})}-\varepsilon_{\mathrm{e}(\mathrm{hh})}}{\varepsilon_{\mathrm{e}(\mathrm{hh})}}}=\tan \left(\sqrt{\frac{m_{\mathrm{CdSe}} \varepsilon_{\mathrm{e}(\mathrm{hh})}}{2}} \frac{L}{\hbar}\right),
$$

where $m_{\mathrm{CdSe}}$ and $m_{\mathrm{CdMgSe}}$ are the effective masses of CdSe QW and CdMgSe barrier, respectively, and $L$ is the CdSe QW thickness.

\section{TABLE}

The experimental and effective mass approximation calculated data on optical transitions in QW CdSe embedded into CdMgSe barriers.

\begin{tabular}{c|c|c|c|c}
\hline \hline$N$ & \multirow{2}{*}{$x$ in CdMgSe } & $L_{Q W C d S e}[\AA]$ & \multicolumn{2}{|c}{$E_{\mathrm{G}}^{\mathrm{eff}}$ CdSe QW } \\
\cline { 4 - 5 } & & & experiment & calculation \\
\hline $\mathrm{A}$ & 0.19 & 53 & 1.824 & 1.814 \\
$\mathrm{~B}$ & 0.17 & 34 & 1.867 & 1.860 \\
$\mathrm{C}$ & 0.18 & 27 & 1.921 & 1.916 \\
$\mathrm{D}$ & 0.16 & 20 & 1.924 & 1.940 \\
$\mathrm{E}$ & 0.14 & 20 & 1.907 & 1.924
\end{tabular}

One can see that there is a good agreement between calculated and experimental data. The small misfits are probably caused by some assumptions we made. Due to the lack of the data on the effective masses, hydrostatic and shear deformation potentials of $\mathrm{MgSe}$ and the relatively small $\mathrm{Mg}$ content in CdMgSe, we employed the values as those for pure CdSe. Taking into account the similarity of 
crystalline properties of the isoelectronic analogs, it has been assumed that elastic constants of MgSe are the same as those of $\mathrm{ZnS}$ [17]. The CdSe parameters used in calculations are taken from Ref. [18].

Good agreement of calculations and experimental data confirms that CdSe embedded into CdMgSe behaves as conventional QW. Additionally, it is confirmed by the observed broadening of the CdSe QW PL peaks with a decrease in the QW thickness, which is typical of two-dimensional QW structures due to enhanced contribution of interface roughness in thinner QWs. This behavior is opposite to that usually observed for highly strained CdSe monolayers in a ZnSe matrix caused by strong strain-induced CdSe layer disordering at larger thickness [2].

In summary, the $\mathrm{CdSe} / \mathrm{CdMgSe} \mathrm{QW}$ heterostructures have been grown for the first time on InAs(001) substrates by molecular beam epitaxy. The dependence of CdMgSe band-gap energy on composition has been determined. The zinc-blende $\mathrm{MgSe}$ band-gap energy and bowing parameter are estimated as $4.05 \mathrm{eV}$ and $0.2 \mathrm{eV}$, respectively. The CdSe QW photoluminescence data are in good agreement with the effective mass approximation calculations.

This work was supported in part by INTAS grant No. 97-31907, the Program of the Ministry of Sciences of RF "Physics of Solid State Nanostructures" grant No. 99-02-17097.

\section{References}

[1] S.V. Ivanov, V.A. Solov'ev, K.D. Moiseev, I.V. Sedova, Ya.V. Terent'ev, A.A. Toropov, B.Ya. Meltzer, M.P. Mikhailova, Yu.P. Yakovlev, P.S. Kop'ev, Appl. Phys. Lett. 78, 1655 (2001).

[2] S.V. Ivanov, A.A. Toropov, T.V. Shubina, S.V. Sorokin, A.V. Lebedev, I.V. Sedova, P.S. Kop'ev, G.R. Pozina, J.P. Bergman, B. Monemar, J. Appl. Phys. 83, 3168 (1998).

[3] F. Firszt, S. Łęgowski, H. Męczyńska, J. Szatkowski, W. Paszkowicz, M. Marczak, J. Cryst. Growth 184/185, 1053 (1998).

[4] A. Ohtake, T. Hanada, K. Arai, T. Komura, S. Miwa, K. Kimura, T. Yasuda, C. Jin, T. Yao, J. Crystal Growth 201/202, 490 (1999).

[5] J.M. Gaines, J. Peruzzello, B. Greenberg, J. Appl. Phys. 73, 2835 (1992).

[6] V.A. Kaygorodov, I.V. Sedova, S.V. Sorokin, O.V. Nekrutkina, T.V. Shubina, A.A. Toropov, S.V. Ivanov, Abstracts of 10th Int. Conf. on II-VI Compounds, Bremen 2001, to be presented.

[7] J. Gutowski, P. Baume, K. Hauke, in: Properties of Wide Bandgap II-VI Semiconductors, Ed. R. Bargava, Inspec IEE, London 1997, p. 37.

[8] C.G. Van de Walle, Phys. Rev. B 39, 1871 (1989).

[9] N. Samarth, H. Luo, J.K. Furdyna, S.B. Qadri, Y.R. Lee, A.K. Ramdas, N. Otsuka, Appl. Phys. Lett. 54, 2680 (1989).

[10] H. Okuyama, K. Nakato, T. Miyajima, K. Akimoto, Jpn. J. Appl. Phys. 2 30, L1620 (1991). 
[11] W.H. Strehlow, E.L. Cook, J. Phys. Chem. Ref. Data 2, 163 (1973).

[12] M.Th. Litz, K. Watanabe, M. Korn, H. Ress, U. Lunz, W. Ossau, A. Waag, G. Landwehr, Th. Walter, B. Neubauer, D. Gerthsen, U. Shussler, J. Cryst. Growth 159, 54 (1996).

[13] V. Pelegrini, R. Atamasov, A. Tredicucci, F. Beltram, C. Amzulini, L. Sobra, L. Vanzetti, A. Francioso, Phys. Rev. B 51, 5171 (1995).

[14] S.-H. Wei, A. Zunger, Appl. Phys. Lett. 72, 2011 (1998).

[15] M.W. Wang, J.F. Swenberg, M.C. Phillips, E.T. Yu, J.O. McCaldin, R.W. Grant, T.C. McGill, Appl. Phys. Lett. 64, 3455 (1994).

[16] M.V. Maximov, I.L. Krestnikov, S.V. Ivanov, N.N. Ledenzov, S.V. Sorokin, Semiconductors 31, 939 (1997).

[17] V.S. Sorokin, S.V. Sorokin, V.A. Kaygorodov, S.V. Ivanov, J. Cryst. Growth 214/215, 130 (2000).

[18] H.J. Lozykowski, V.K. Shastri, J. Appl. Phys. 69, 3235 (1991). 